\section{(C) OPEN ACCESS}

\title{
Testing bioresorbable stent feasibility in a rat aneurysm model
}

\author{
Basil Erwin Grüter, ${ }^{\oplus 1,2}$ Dominik Täschler, ${ }^{2}$ Fabio Strange, ${ }^{1,2}$ Jeannine Rey, ${ }^{2}$ \\ Michael von Gunten, ${ }^{3}$ Denis Grandgirard, ${ }^{4,5}$ Stephen L Leib, ${ }^{4,5}$ Luca Remonda, ${ }^{6}$ \\ Hans Rudolf Widmer, ${ }^{5,7}$ Edin Nevzati, ${ }^{2}$ Javier Fandino, ${ }^{1,2}$ Serge Marbacher, ${ }^{1,2}$ \\ Daniel Coluccia ${ }^{1,2}$
}

\begin{abstract}
- Additional material is published online only. To view please visit the journal online (http://dx.doi.org/10.1136/ neurintsurg-2018-014697).

1 Department of Neurosurgery, Kantonsspital Aarau, Switzerland

${ }^{2}$ Cerebrovascular Research Group, Department for Biomedical Research (DBMR), Universitiy of Bern, Switzerland ${ }^{3}$ Institute of Pathology Laenggasse, Ittigen, Switzerland ${ }^{4}$ Neuroinfection Laboratory, Institute for Infectious Diseases, University of Bern, Switzerland ${ }^{5}$ Cluster for Regenerative Neuroscience, Department for Biomedical Research (DBMR), Universitiy of Bern, Switzerland ${ }^{6}$ Division of Neuroradiology, Department of Radiology, Kantonsspital Aarau, Switzerland

${ }^{7}$ Department of Neurosurgery, Inselspital Bern, Switzerland
\end{abstract}

Correspondence to Dr Basil Erwin Grüter, Department of Neurosurgery, c/o NeuroResearch Office, Kantonsspital Aarau, Tellstrasse 1, 5001 Aarau, Switzerland; basil.grueter@ksa.ch

Received 27 December 2018 Revised 9 February 2019 Accepted 22 February 2019

Check for updates

(C) Author(s) (or their employer(s)) 2019. Re-use permitted under CC BY-NC. No commercial re-use. See rights and permissions. Published by BMJ.

To cite: Grüter $\mathrm{BE}$, Täschler D, Strange F, et al. J Neurolntervent Surg Epub ahead of print: [please include Day Month Year]. doi:10.1136/

neurintsurg-2018-014697

\section{ABSTRACT}

Background Advances in stent-assisted coiling have incrementally expanded endovascular treatment options for complex cerebral aneurysms. After successful coil consolidation and aneurysm occlusion, endovascular scaffolds are no longer needed. Thus, bioresorbable stents that disappear after aneurysm healing could avoid future risks of in-stent thrombosis and the need for lifelong antiplatelet therapy.

Objective To assess the applicability and compatibility of a bioresorbable magnesium- alloy stent (brMAS) for assisted coiling.

Methods Saccular sidewall aneurysms were created in 84 male Wistar rats and treated with brMAS alone, brMAS + aspirin, or brMAS + coils + aspirin. Control groups included no treatment (natural course), solely aspirin treatment, or conventional cobaltchromium stent + coils + aspirin treatment. After 1 and 4 weeks, aneurysm specimens were harvested and macroscopically, histologically, and molecularly examined for healing, parent artery perfusion status, and inflammatory reactions. Stent degradation was monitored for up to 6 months with micro-computed and optical coherence tomography.

Results Aneurysms treated with brMAS showed advanced healing, neointima formation, and subsequent stent degradation. Additional administration of aspirin sustained aneurysm healing while reducing stent-induced intraluminal and periadventitial inflammatory responses. No negative interaction was detected between platinum coils and brMAS. Progressive brMAS degradation was confirmed.

Conclusions brMAS induced appropriate healing in this sidewall aneurysm model. The concept of using bioresorbable materials to promote complete aneurysm healing and subsequent stent degradation seems promising. These results should encourage further device refinements and clinical evaluation of this treatment strategy for cerebrovascular aneurysms.

\section{BACKGROUND}

Endovascular coiling techniques for intracranial aneurysms have continuously improved since their introduction in the 1990s. ${ }^{1}$ However, considerable aneurysm reperfusion rates (up to $30 \%$ ) and inapplicability for wide-necked aneurysms remain significant limitations of conventional coiling. ${ }^{2-4}$ Stent-assisted coiling helped to overcome some of these challenges. First introduced in 1997, this technique combined a balloon-mounted coronary stainless steel stent with Guglielmi detachable coils. ${ }^{5}$ The stent acts as a bridging scaffold over the aneurysm neck and prevents coil migration. It simultaneously allows higher coil packing and provides an endoluminal matrix for endothelial growth, which ultimately decreases aneurysm reperfusion rates. ${ }^{6}$ A major disadvantage of stent implantation is the increased risks of local thrombus formation and ischemic stroke that necessitate lifelong antiplatelet therapy. This may lead to side effects without completely eliminating the risks of late stent thrombosis and in-stent stenosis. ${ }^{7}$ Permanent stents may also cause an intraparenchymal foreign-body reaction, ${ }^{89}$ which, along with the implanted inert coils, can impede complete aneurysm healing. The endovascular scaffold becomes unnecessary after coil integration and aneurysm healing. Devices that resorb after coil-induced thrombus organization and complete aneurysm occlusion could obviate the risks associated with a lasting stent. Recent advances in material science have enabled the development of bioresorbable vascular implants composed of polyL-lactic acid and a magnesium alloy. ${ }^{10}$ Bioresorbable stent use has already been studied in clinical trials for stenotic coronary artery disease. ${ }^{11}{ }^{12}$ Our study aimed to investigate the impact of a bioresorbable magnesium-alloy stent (brMAS) on early aneurysm remodelling in a rat sidewall saccular aneurysm model.

\section{METHODS}

\section{Study design}

Decellularized sidewall saccular aneurysms were created in 84 male Wistar rats that underwent subsequent treatments: brMAS alone $(n=23)$, brMAS + aspirin $(\mathrm{n}=19)$, brMAS + coils + aspirin $(n=7)$, aspirin alone $(n=6)$, conventional cobaltchromium stent $(\mathrm{CoCrS})+$ coils + aspirin $(n=6)$, and no treatment $(\mathrm{n}=23)$. Aneurysm healing, perfusion status of the underlying vessel, and inflammatory reactions were examined and compared among the six groups. Magnesium-alloy stent degradation was tracked monthly for 6 months (figure 1).

\section{Animals}

Animals were aged $\geq 12$ weeks, weighed $567 \pm 73$ g, and received care in accordance with institutional 


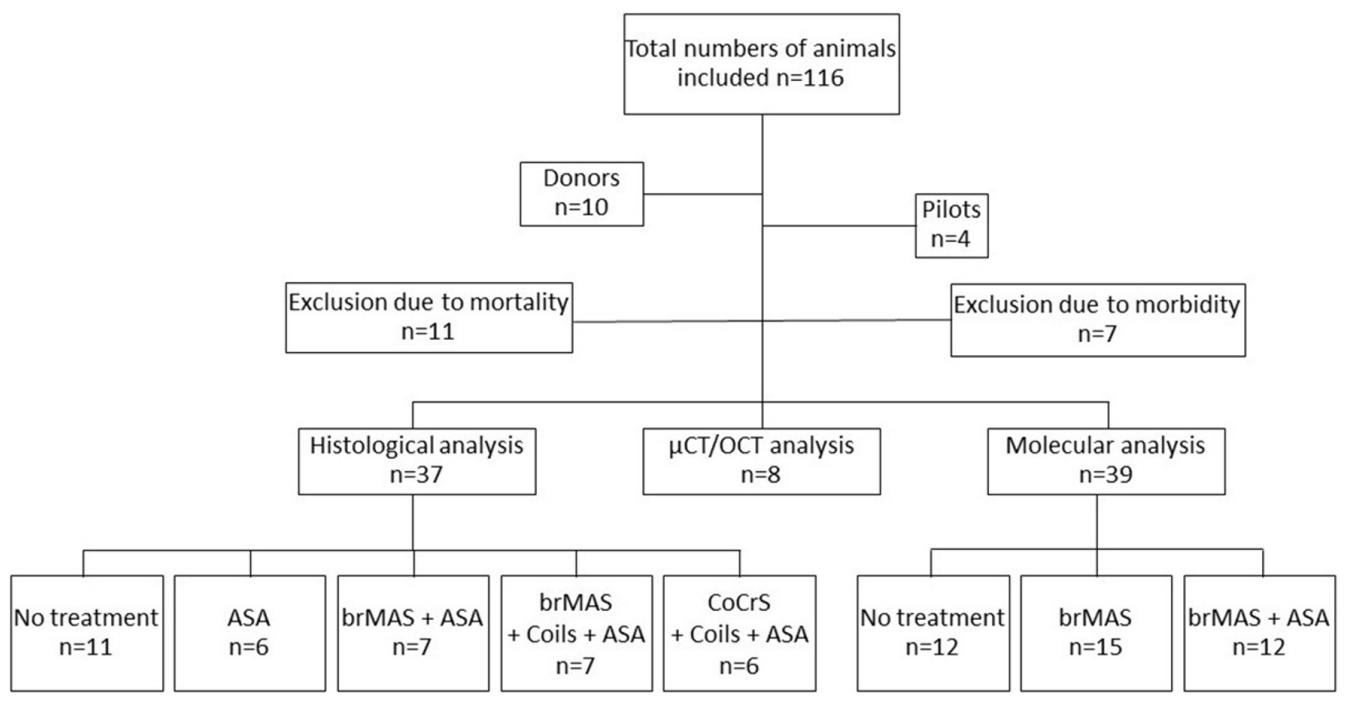

Figure 1 Study flowchart showing the numbers of animals in each experimental condition. Exclusions from the final analysis were deaths caused by subacute hemorrhage $(n=4)$, anesthesia-related problems $(n=1)$, acute in-stent thrombosis $(n=1)$, failure of stent deconvolution ( $n=1)$, pulmonary embolism $(n=1)$, and unknown $(n=3)$. Exclusions due to morbidity necessitating premature euthanasia were anesthesia-related problems $(n=3)$, subacute in-stent thrombosis $(n=2)$, intestinal perforation $(n=1)$, and unknown $(n=1)$. ASA, aspirin; brMAS, bioresorbable magnesium-alloy stent; $\mathrm{CoCrS}$, permanent cobalt-chromium stent; $\mu \mathrm{CT}$, micro-CT; OCT, optical coherence tomography.

guidelines. Experiments were approved by the Committee for Animal Care of the Canton Bern, Switzerland (BE 65/16). In accordance with ARRIVE (Animal Research: Reporting of In Vivo Experiments) guidelines, ${ }^{13}$ an a priori power analysis was performed to calculate sample sizes for experimental subgroups.

\section{Aneurysm formation and stent placement}

After anesthesia induction by inhalation of isoflurane $4 \%$, an intraperitoneal injection of $0.5 \mathrm{mg} / \mathrm{kg}$ medetomidine (Medetor, Virbac, Switzerland) and $50 \mathrm{mg} / \mathrm{kg}$ ketamine hydrochloride (Ketalar, Pfizer, Switzerland) was administered. As previously described, sidewall aneurysms were created by end-to-side anastomosis of an arterial vessel pouch from a donor animal (thoracic aorta) to the clamped abdominal aorta of a recipient animal. All grafts had previously been decellularized by incubation in $0.1 \%$ sodium dodecyl sulfate for 10 hours at $37^{\circ} \mathrm{C} .{ }^{14} \mathrm{~A}$ digital video camera (Sony NEX-5R, Sony, Tokyo, Japan) attached to a stereo microscope (OPMI, Carl Zeiss AG, Oberkochen, Germany) was used to document aneurysm dimensions preoperatively, at harvest, and during operative procedures. With aneurysm formation, the abdominal aorta was cannulated below the iliolumbar artery, and the stent was introduced through an intravasal lock and dilatator using a modified Seldinger technique. ${ }^{15}$ The two devices were a magnesium-alloy stent coated with degradable poly-L-lactic acid polymer (Biotronik, Bülach, Switzerland), $6 \mathrm{~mm}$ length (2.5 $\mathrm{mm}$ diameter), and nominal pressure of $8 \mathrm{bar}$; and a conventional CoCrS (Pro-Kinetic Energy, Biotronik), $9 \mathrm{~mm}$ length (2.5 $\mathrm{mm}$ diameter), and nominal pressure of 9 bar. A target helical ultra-coil (Stryker, Kalamazoo, Michigan, USA) of $2 \mathrm{~cm}$ length ( $2 \mathrm{~mm}$ diameter) was used for coiling. Aspirin (Alcacyl 500, Hänseler AG, Switzerland) was administered weight-adapted via restriction of these animals to aspirin-enriched drinking water $(2000 \mathrm{mg} / \mathrm{L})$.

\section{Fluorescence angiography, macroscopic inspection, and tissue preparation}

A laparotomy was performed for aneurysm dissection at the specified follow-up time. Fluorescence angiography was performed to assess dynamic perfusion status after intravenous injection of
$2 \mathrm{~mL}$ fluorescein (fluorescein 10\% Faure, $0.5 \mathrm{~g} / 5 \mathrm{~mL}$ ), followed by illumination (light source 465 to $490 \mathrm{~nm}$ ) of the aneurysm, which was filmed with a specified detection filter as previously described. ${ }^{16}$ After animals were euthanized by overdosing with intracardial ketamine hydrochloride (Narketan, $120 \mathrm{mg} /$ $\mathrm{kg}$ ketamine injection, Vetoquinol, Switzerland), the aneurysm was harvested and measured in all dimensions, and the posterior aorta was opened to inspect the aneurysm orifice. Tissues were immediately shock frozen on dry ice for molecular analysis or fixed in formalin (4\% weight/volume solution, J.T. Baker, Arnhem, The Netherlands) until embedded in paraffin for histological analysis.

\section{Post mortem analysis}

Gelatine sodium dodecyl sulfate-polyacrylamide gel electrophoresis zymography was performed to detect the enzymatic activity of matrix metalloproteinase (MMP)-2 and -9. Protein homogenates $(5 \mu \mathrm{g})$ were submitted to electrophoresis under non-reducing conditions in polyacrylamide gels containing type A gelatine from porcine skin $(1 \% \mathrm{v} / \mathrm{v})$. The assessment of cytokines/ chemokines or other analytes was performed using a rat magnetic Luminex assay 7-Plex kit (R\&D Systems, Minneapolis, Minnesota, USA), according to the manufacturer instructions. Concentrations of the different analytes were determined in $100 \mu \mathrm{g}$ of crude protein homogenates using Bio-Plex Manager Software 4.1.1 (Bio-Rad Laboratories, Hercules, California, USA) with a 5-parameter logistic curve-fitting method. The histological study included hematoxylin-eosin, Masson-Goldner trichrome, smooth muscle actin, and von Willebrand factor (F8) staining. Further post mortem analyses included optical coherence tomography (OCT) and micro-computed tomography $(\mu \mathrm{CT})$ imaging of the aneurysm and underlying vessels. A detailed description of the methods is given in the online supplementary data.

\section{Statistical analysis}

Statistical analysis was performed with Graph Pad Prism 7 (version 7.02, GraphPad software, San Diego, California, USA). Linear regression analysis was used to compare healing status (complete vs residual perfusion). Numeric samples of two groups 


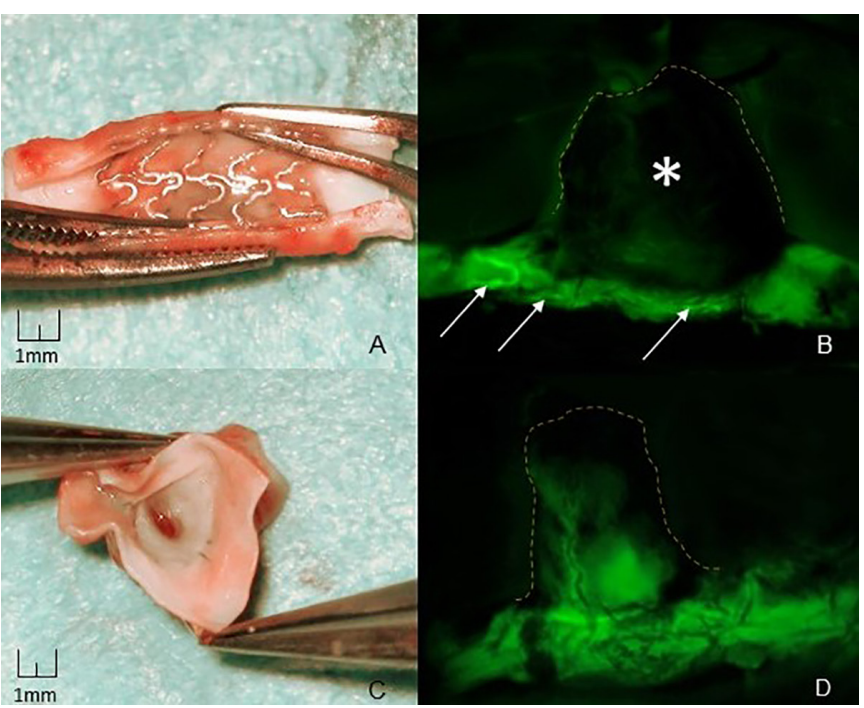

Figure 2 Comparison of a completely healed aneurysm after stent treatment and a poorly healed untreated aneurysm. Gross complete healing was noted after opening the parent artery opposite the aneurysm (A), and fluorescence angiography (B) confirmed a continuous neointima over the former orifice with no residual perfusion inside the aneurysm (*) after treatment with a bioresorbable magnesium alloy stent (arrows). Poor healing in macroscopic (C) and fluorescence angiographic (D) views of a 28-day-old untreated aneurysm.

were compared with Mann-Whitney U tests for non-parametrically distributed values and Student t-tests for parametrically distributed values. One-way analyses of variance followed by Dunn's multiple comparison tests were used to study heterogeneity in multiple groups. Values are expressed as median and IQR or mean \pm SD. A p value $<0.05$ was considered statistically significant.

\section{RESULTS \\ brMAS highlights}

Aneurysms treated with brMAS showed pronounced healing, strong neointima formation, and stent degradation. At the 4-week follow-up, fluorescence angiography and macroscopic inspection showed complete aneurysm occlusion in 22 of 26 brMAS stent-treated animals compared with 4 of 20 animals without stents $(p<0.0001$, figure 2$)$. The endothelium-free aneurysm orifice showed a strong neointima after brMAS stenting, which prevented aneurysm perfusion. Histological and molecular analyses showed increased locoregional inflammation in stent-treated animals compared with untreated. Aspirin significantly reduced inflammation in stent-treated animals as observed in histological assessments and based on inflammatory protein expression. Qualitative $\mu \mathrm{CT}$ and OCT revealed progressive degradation of brMAS; we observed fractures and clefts in the stent loop integrity and progressive rarefication of metal content in the vessel wall (figure 3).

\section{Aneurysm remodeling and inflammatory response}

Microscopic evaluation confirmed advanced healing with neointima formation but expectedly higher inflammation after brMAS treatment compared with no stent: significantly more neutrophils accumulated in the thrombi of aneurysms with stent treatment than in those without stents $(p=0.028)$. Aspirin administration in animals with stents significantly reduced aneurysm wall inflammation $(\mathrm{p}=0.02)$ and yielded better neointima

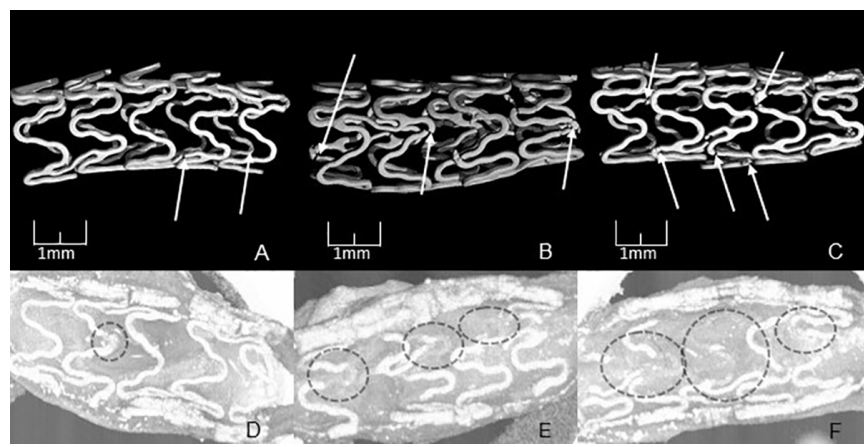

Figure 3 Micro-CT $(\mu \mathrm{CT}) 3 \mathrm{D}$ reconstruction (upper row) and optical coherence tomography (OCT) reconstruction (lower row) showing progressive stent degradation after 1 month (A and D), 3 months ( $B$ and $E$ ), and 4 months ( $C$ and $F$ ) follow-up. $\mu C T$ shows progressive loss of scaffold integrity (arrows). Degradation of magnesium (circles) is best seen on OCT because the laser is reflected on magnesium but penetrates temporary calcium phosphate deposits that resulted from stent degradation; this has a density similar to magnesium and can therefore not be distinguished on $\mu \mathrm{CT}$.

formation $(\mathrm{p}=0.02)$, with no significant difference in inflammatory cells in the thrombi. Online supplementary figure I shows the detailed histological analysis. The increased inflammatory reaction after stent placement was confirmed by quantitative molecular analyses. Luminex analysis of proinflammatory cytokines (interleukin [IL] -6 and IL-1 $\beta$ ) and gelatine zymography for matrix-degrading MMP-9 showed that concentrations were highest after 1 week of aneurysm treatment and decreased at week 4 (all values given in the online supplementary table). Aspirin administration effectively reduced levels of proinflammatory cytokines in stent-treated animals in the acute period (week 1) Concentrations of IL-1 $\beta(p=0.01)$, IL-6 $(p=0.045)$, MMP-9 ( $\mathrm{p}=0.009)$, and MMP-2 $(\mathrm{p}=0.01)$ were significantly lower after 1 week in brMAS + aspirin treated animals compared with brMAS treatment without aspirin. In the chronic phase of aneurysm healing (week 4), there were no significant differences in inflammation marker levels in the brMAS + aspirin and untreated groups.

\section{Stent-assisted coiling with brMAS}

No macroscopic or histological signs of negative interactions were detected between the platinum coils and brMAS, such as increased cellular reactions, material failure, or tissue necrosis. Furthermore, there were no differences in aneurysm healing between coiled aneurysms with brMAS or CoCrS (example of histology shown in figure 4, analysis in online supplementary figure II). Except for one CoCrS-treated case, all aneurysms treated by brMAS or CoCrS + coils showed complete healing with coherent neointima formation and no residual perfusion after 4 weeks. No differences were observed between the two stent groups for periadventitial inflammation $(p>0.99)$, aneurysm wall inflammation $(p>0.99)$, or neutrophils in the thrombus $(\mathrm{p}>0.99)$.

\section{Mid-term and long-term findings and stent degradation}

Preserved aneurysm healing with no residual perfusion was observed at monthly follow-up examinations from 1 to 6 months. Complete stent endothelialization was achieved, and the mean neointima thickness varied from $137.26 \mu \mathrm{m}$ at 1 month, $75.33 \mu \mathrm{m}$ at 2 months, $66.99 \mu \mathrm{m}$ at 3 months, $97.87 \mu \mathrm{m}$ at 4 months, and $80.17 \mu \mathrm{m}$ at 6 months, as assessed by OCT (see 


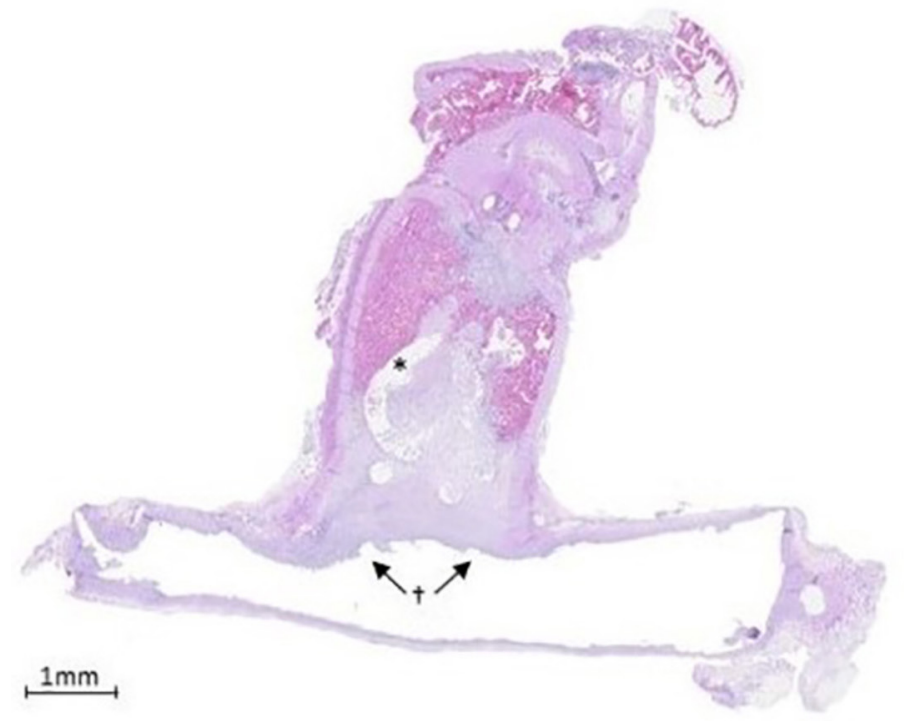

A

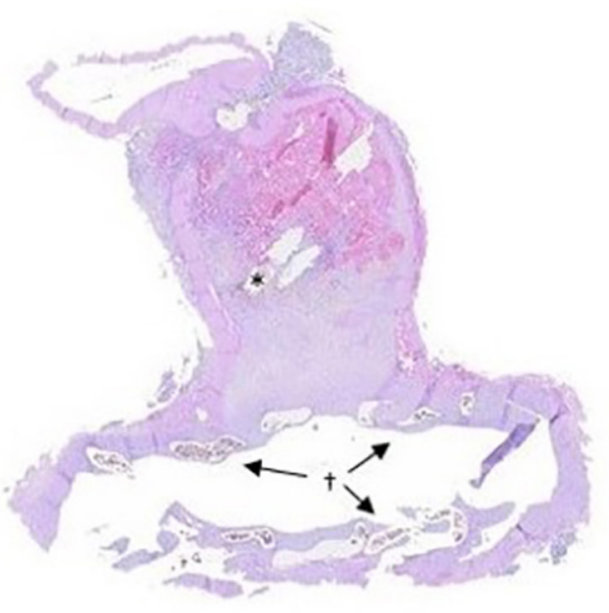

B

Figure 4 Representative histologic images of aneurysms treated with a cobalt-chromium stent (A) and bioresorbable magnesium-alloy stent (B) after 28 days. Treatment with either stent induced strong neointima formation with a thick cell layer over the former aneurysm orifice and advanced thrombus organization. * Coil artifact; tstent artifact.

onlinesupplementary figure III). No systemic or in situ comorbidities or negative side effects of brMAS were detected on any follow-up examination. In particular, we did not detect intraluminal debris with progressive stent degradation. Qualitative $\mu \mathrm{CT}$ and OCT investigations showed progressive brMAS degradation characterized by fractures and clefts in the stent loop integrity and progressive rarefication of metal content in the vessel wall (figure 3).

\section{DISCUSSION}

Our study aimed to evaluate the histopathological effects of a contemporary brMAS on early aneurysm remodeling to assess whether its material and design properties are suitable for further research efforts. Bioresorbable magnesium-alloy stents prompted adequate aneurysm healing responses in the rat model, and no negative interactions between platinum coils and brMAS were detected in the histological analysis.

Although brMAS is clinically approved for coronary artery disease, cerebrovascular applications are relatively new. To the best of our knowledge, only two studies have investigated the use of bioresorbable stents for aneurysm treatment. Extended analysis of interaction with thrombus formation, vessel wall remodeling, and data on combined use with coils are lacking. Using endovascular placement in a rabbit aneurysm model, Wang et $a l^{17}$ reported effective aneurysm occlusion in all 12 of the animals treated with magnesium-alloy stents. Similarly, Wang et $a l^{18}$ reported effective and safe treatment of carotid artery aneurysms in 13 rabbits using bioresorbable magnesium flow diverters. We confirmed good rates of aneurysm healing with brMAS treatment in our rat model.

In addition, our study provides new data for endothelium-free aneurysm orifice remodeling, inflammation processes, and stent-coil interactions. Besides the provided mechanical barrier for coils, stents promote neointimal proliferation and serve as scaffolds for cells that will eventually form a neointimal layer that changes hemodynamics and facilitates aneurysm occlusion. ${ }^{19}$ This is important, because aneurysms with rarefication of mural cells are prone to growth and rupture. ${ }^{20}$ Owing to their decreased cellularity, these aneurysms exhibit reduced ability for aneurysm healing and neointima forming, as aneurysm-organizing cells are mainly recruited from the adjacent parent vessel. $^{2122}$ Accordingly, stent placement will help these cells to settle down along the scaffold, and, particularly, also along the aneurysm orifice.

Locoregional inflammation induced by intravascular implants is of concern as it may induce critical, rupture-prone alterations of the aneurysm wall, especially in cases where subsequent coil implantation might fail. On the other hand, inflammation at the injured endothelium and media layers is essential in aneurysm healing. It activates vascular smooth muscle cells to proliferate and phenotypically switch from contractile to synthetic cells that synthesize a matrix to support aneurysm cicatrization and neointima formation. ${ }^{23-25}$ However, an overwhelming inflammatory reaction may promote aneurysm formation, growth, and rupture. ${ }^{26}$ Pronounced inflammation after stent implantation should therefore be controlled to promote adequate healing. We observed that aspirin significantly reduced inflammation after stent implantation (particularly in the vulnerable first weeks), and aneurysm treatment with brMAS + coils and aspirin achieved fast aneurysm healing, strong neointima formation, aneurysmal scarification without residual perfusion, and subsequent stent degradation. Most clinically used stents are pharmaceutically coated with an immunosuppressant (ie, sirolimus). We used stents without an immunosuppressive coating to reduce potential interference in our analysis of material-tissue interactions.

Lifelong antiplatelet medication is essential to avoid in-stent thrombosis and thromboembolic events. Tissues are particularly vulnerable immediately after stent implantation. The early thrombosis rate may even be higher for current bioresorbable stents than with conventional metal stents; therefore, antiplatelet treatment remains essential in the acute phase. ${ }^{27}$ However, following neointima formation and aneurysmal scarification, the adverse effects of long-term aspirin use prevail. ${ }^{28}$ In an alternative approach to overcome drawbacks of systemic (dual) antiplatelet therapy, a recent study suggested phosphorylcholine 
surface modification of flow diverters to reduce platelet-specific thrombogenicity. ${ }^{29}$ The rationale for using brMAS is that complete stent degradation would allow for complete cessation of an antiplatelet regimen.

Bioresorbable magnesium-alloy stents functioned well in combination with conventional uncoated platinum coils. As balloon inflation itself will induce some degree of endothelial damage and thus interfere with aneurysm remodeling activities, as a first step we chose to compare brMAS-induced endothelialization and aneurysm healing processes with those following use of a contemporary balloon-expandable CoCrS. In addition, CoCrSs are known for favorable endovascular biocompatibility versus other metal compositions. ${ }^{30}$ Notably, brMAS performed comparably to the latest generation of CoCrS. No relevant differences were observed in neointima formation, orifice obliteration, aneurysm healing, or inflammatory reaction. However, we did not formally assess the contribution of differences in stent design (eg, mesh forms, size, length) to the results. The relevance of scaffold design remains unclear, as a previous study which compared different flow-diverter designs in a rabbit aneurysm model reported temporal and spatial dependency of endothelialization on scaffold design, but no difference in final aneurysm occlusion. ${ }^{22}$

Whereas the first stent-assisted coiling treatments used balloon-expandable coronary stainless-steel stents, modern neurovascular stents are commonly made of nitinol. Their superelastic and shape memory properties allow for self-expandable devices, facilitating gentle stent deployment, which reduces the risk of endothelial damage. Another favorable feature of contemporary neurovascular nitinol stents is their thin strut thickness $(<100 \mu \mathrm{m})$. In stenotic coronary artery disease, increasing strut thickness correlates with the restenosis rate. ${ }^{31}$ Nitinol stents of the same strut thickness offer less radial force than cobalt-chromium, which is why nitinol stents are not widely used in coronary disease, where stents need to hold against stenotic atherosclerotic vessels. ${ }^{32}$ Strength properties are less critical in assisted coiling; treatment is not directed towards an atherosclerotic vessel, which is why lowering strut thickness is appropriate. In contrast, current brMAS are produced using a weaving technique that affects the minimal achievable strut thickness (the brMAS strut thickness used was $120 \mu \mathrm{m}$ ), making balloon expansion necessary.

While stent-treated aneurysms remained obliterated over the mid- and long-term follow-up, we confirmed continuous brMAS degradation over time. This is consistent with data from previous studies in a porcine heart model, which showed more than two-thirds degradation after 6 months, over 95\% degradation after 1 year and nearly complete degradation (99.8\%) after 2 years of a stent that was $3 \mathrm{~mm}$ longer and sirolimus covered, but otherwise identical to the scaffold we used. ${ }^{33} 34$ The relatively rapid degradation of early magnesium stents represents a main limitation in atherosclerotic coronary arteries, where longer mechanical support is desirable. However, fast degradation may be advantageous in neurovascular indications, as coils need only short-term support until a neointima is formed. Furthermore, brMAS degradation rates are modifiable, depending on the alloy mixture, stent dimensions, and coverings of the stent. ${ }^{12}$ During bioresorbable stent degradation, neointimal thickness may initially increase before reaching stable luminal dimensions. ${ }^{17} 35$ Our series confirmed neointima remodeling during the first few months with a subsequent steady state of thickness after 5 months.

Although our preclinical findings show promising brMASaneurysm interactions, specific stent adaptations and refinements are needed to meet the requirements for eventual clinical neurovascular applications. Nevertheless, the field of bioresorbable endovascular materials is evolving and could create new treatment strategies for cerebrovascular diseases.

\section{Limitations}

Our investigation of brMAS for aneurysm therapy focused on the acute and early chronic phases but did not examine longterm effects. No late in-stent stenosis occurred during the 6 -month study period. Other limitations are associated with the aneurysm model itself: surgical aneurysm creation substantially affects inflammation and healing. We minimized this by choosing appropriate follow-up time points. Furthermore, surgical aneurysm creation and endovascular treatment were performed at the same time to reduce animal distress and increase survival. This setting did not allow for digital subtraction angiography; evaluation of the perfusion status of the aneurysm and underlying vessel was based on in-vivo fluorescence angiography and post mortem macroscopic and histologic evaluation. In addition, our study focused on the suitability of brMAS in aneurysmatic disease, and on compatibility of brMAS with coils. In a more advanced state, a bioresorbable stent specifically optimized for stent-assisted coiling should also be evaluated in the light of dual antiplatelet standard medication. Lastly, aneurysm creation was standardized across animals, but sutured aneurysms in rats obviously lack some properties of naturally formed cerebral aneurysms (eg, sidewall position, exposure to non-physiological blood flow, tissue environment). Better understanding of brMAS performance in cerebral aneurysms will be achieved by testing in a rabbit bifurcation model that exhibits similar flow dynamics to those of cerebral aneurysms and allows for angiographic imaging and distant endovascular stent deployment.

\section{CONCLUSION}

In conclusion, brMAS treatment was feasible and successful in our rat sidewall model with adequate aneurysm remodeling. Pronounced inflammation triggered by the bioresorbable scaffold was markedly reduced by administration of aspirin. Using bioresorbable materials to enable complete healing with subsequent degradation of foreign materials is a promising concept that could be developed in the clinical setting of cerebrovascular disease.

Acknowledgements We acknowledge Eugen Hofmann and Philine Zumstein for their support with magnesium stents. We thank Franziska Simon, Mark Siegrist, and Stefano di Santo for their excellent technical assistance. We also thank Rainer Grobholz for his support with digital histology. We thank Mary Kemper and Lindsay Reese for editing. Finally, we thank Carline Perren for her help with regulatory and administrative tasks.

Contributors BEG and DC conceived the study. BEG, DT, FS, JR, EN, MvG, DG, $S L L, H R W$, and DC conducted the experiments and acquired data. Analysis and interpretation of data was performed by BEG, DT, DG, LR, JF, SM, and DC. Statistical analysis was performed by BEG, HRW, SM, and DC. BEG took the lead in writing the manuscript with critical feedback from all authors. JF, LR, and SM supervised the project and DC was in charge of the overall direction and planning. All authors approved the final version to be published and agree to be accountable for all aspects of the work.

Funding This work was supported by research funds of the Research Council, Kantonsspital Aarau, Aarau, Switzerland (FR 1400.000.054). The bioresorbable magnesium alloy stents and cobalt-chromium stents were supplied by Biotronik AG, Centre for Vascular Intervention, Bülach, Switzerland. The authors are solely responsible for the design and conduct of the presented study and declare no competing interests.

Competing interests None declared.

Patient consent for publication Not required.

Provenance and peer review Not commissioned; externally peer reviewed.

Open access This is an open access article distributed in accordance with the Creative Commons Attribution Non Commercial (CC BY-NC 4.0) license, which 
permits others to distribute, remix, adapt, build upon this work non-commercially, and license their derivative works on different terms, provided the original work is properly cited, appropriate credit is given, any changes made indicated, and the use is non-commercial. See: http://creativecommons.org/licenses/by-nc/4.0/.

\section{REFERENCES}

1 Bender MT, Wendt $\mathrm{H}$, Monarch T, et al. Shifting treatment paradigms for ruptured aneurysms from open surgery to endovascular therapy over 25 years. World Neurosurg 2017;106:919-24.

2 Nieuwkamp DJ, Setz LE, Algra A, et al. Changes in case fatality of aneurysmal subarachnoid haemorrhage over time, according to age, sex, and region: a metaanalysis. Lancet Neuro/ 2009;8:635-42.

3 Songsaeng D, Geibprasert S, ter Brugge KG, et al. Impact of individual intracranial arterial aneurysm morphology on initial obliteration and recurrence rates of endovascular treatments: a multivariate analysis. J Neurosurg 2011;114:994-1002.

4 Li H, Pan R, Wang H, et al. Clipping versus coiling for ruptured intracranial aneurysms: a systematic review and meta-analysis. Stroke 2013;44:29-37.

5 Higashida RT, Smith W, Gress D, et al. Intravascular stent and endovascular coil placement for a ruptured fusiform aneurysm of the basilar artery. Case report and review of the literature. J Neurosurg 1997;87:944-9.

6 Alfke K, Straube T, Dörner L, et al. Treatment of intracranial broad-neck aneurysms with a new self-expanding stent and coil embolization. AJNR Am J Neuroradiol 2004:25:584-91.

7 Riedel CH, Tietke M, Alfke K, et al. Subacute stent thrombosis in intracranial stenting. Stroke 2009:40:1310-4.

8 Minks D, Briley D, Schulz U, et al. Suspected cerebral foreign body granuloma following endovascular treatment of intracranial aneurysm: imaging features. Neuroradiology 2015;57:71-3.

9 Lorentzen AO, Nome T, Bakke SJ, et al. Cerebral foreign body reaction after carotid aneurysm stenting. Interv Neuroradiol 2016;22:53-7.

10 Wiebe J, Nef HM, Hamm CW. Current status of bioresorbable scaffolds in the treatment of coronary artery disease. J Am Coll Cardiol 2014;64:2541-51.

11 Erbel R, Di Mario C, Bartunek J, et al. Temporary scaffolding of coronary arteries with bioabsorbable magnesium stents: a prospective, non-randomised multicentre trial. Lancet 2007;369:1869-75.

12 Haude M, Ince H, Abizaid A, et al. Sustained safety and performance of the secondgeneration drug-eluting absorbable metal scaffold in patients with de novo coronary lesions: 12-month clinical results and angiographic findings of the BIOSOLVE-II firstin-man trial. Eur Heart J 2016;37:2701-9.

13 Kilkenny C, Browne WJ, Cuthill IC, et al. Improving bioscience research reporting: the ARRIVE guidelines for reporting animal research. PLOS Biol 2010;8:e1000412.

14 Marbacher S, Marjamaa J, Abdelhameed E, et al. The Helsinki rat microsurgical sidewall aneurysm model. J Vis Exp 2014:e51071.

15 Nevzati E, Rey J, Coluccia D, et al. Biodegradable magnesium stent treatment of saccular aneurysms in a rat model - introduction of the surgical technique. J Vis Exp 2017; 128.

16 Grüter BE, Täschler D, Rey J, et al. Fluorescence video sngiography for evaluation of dynamic perfusion status in an aneurysm preclinical experimental setting. Operative Neurosurgery. In press. 2018.
17 Wang W, Wang YL, Chen M, et al. Magnesium alloy covered stent for treatment of a lateral aneurysm model in rabbit common carotid artery: an in vivo study. Sci Rep 2016;6:37401

18 Wang K, Yuan S, Zhang X, et al. Biodegradable flow-diverting device for the treatment of intracranial aneurysm: short-term results of a rabbit experiment. Neuroradiology 2013;55:621-8.

19 Wanke I, Forsting M. Stents for intracranial wide-necked aneurysms: more than mechanical protection. Neuroradiology 2008;50:991-8.

20 Marbacher S, Marjamaa J, Bradacova K, et al. Loss of mural cells leads to wall degeneration, aneurysm growth, and eventual rupture in a rat aneurysm model. Stroke 2014;45:248-54.

21 Frösen J, Marjamaa J, Myllärniemi $M$, et al. Contribution of mural and bone marrow-derived neointimal cells to thrombus organization and wall remodeling in a microsurgical murine saccular aneurysm model. Neurosurgery 2006;58:936-44.

22 Marosfoi M, Langan ET, Strittmatter L, et al. In situ tissue engineering: endothelial growth patterns as a function of flow diverter design. J Neurointerv Surg 2017;9:994-8

23 Frösen J, Tulamo R, Paetau A, et al. Saccular intracranial aneurysm: pathology and mechanisms. Acta Neuropathol 2012;123:773-86.

24 Chalouhi N, Ali MS, Jabbour PM, et al. Biology of intracranial aneurysms: role of inflammation. J Cereb Blood Flow Metab 2012;32:1659-76.

25 Newby AC, Zaltsman AB. Molecular mechanisms in intimal hyperplasia. J Pathol 2000;190:300-9.

26 Chalouhi N, Hoh BL, Hasan D. Review of cerebral aneurysm formation, growth, and rupture. Stroke 2013;44:3613-22.

27 Kereiakes DJ, Onuma Y, Serruys PW, et al. Bioresorbable vascular scaffolds for coronary revascularization. Circulation 2016;134:168-82.

28 Li L, Geraghty OC, Mehta Z, et al. Age-specific risks, severity, time course, and outcome of bleeding on long-term antiplatelet treatment after vascular events: a population-based cohort study. Lancet 2017;390:490-9.

29 Hagen MW, Girdhar G, Wainwright J, et al. Thrombogenicity of flow diverters in an ex vivo shunt model: effect of phosphorylcholine surface modification. J Neurointerv Surg 2017:9:1006-11.

30 Jeewandara TM, Wise SG, Ng MKC. Biocompatibility of coronary stents. Materials 2014; 7:769-86.

31 Kastrati A, Mehilli J, Dirschinger J, et al. Intracoronary stenting and angiographic results: strut thickness effect on restenosis outcome (ISAR-STEREO) trial. Circulation 2001;103:2816-21.

32 Lu H, De Winter RJ, Koch KT. The STENTYS self-apposing stent technology in coronary artery disease: literature review and future directions. Expert Rev Med Devices 2018;15:479-87.

33 Joner M, Ruppelt $\mathrm{P}$, Zumstein $\mathrm{P}$, et al. Preclinical evaluation of degradation kinetics and elemental mapping of first- and second-generation bioresorbable magnesium scaffolds. Eurolntervention 2018;14:e1040-8.

34 Haude $M$, Ince $H$, Abizaid A, et al. Safety and performance of the second-generation drug-eluting absorbable metal scaffold in patients with de-novo coronary artery lesions (BIOSOLVE-II): 6 month results of a prospective, multicentre, non-randomised, first-in-man trial. Lancet 2016:387:31-9.

35 Waksman R, Zumstein P, Pritsch M, et al. Second-generation magnesium scaffold Magmaris: device design and preclinical evaluation in a porcine coronary artery model. Eurolntervention 2017;13:440-9. 